\title{
Pregnancy Complications and Risk of Uterine Rupture Among Women With Singleton Pregnancies in China
}

\section{Jing Tao}

National Office for Maternal and Child Health Surveillance of China, West China Second University Hospital, Sichuan University, Chengdu, Sichuan, China

\section{Yi Mu}

National Office for Maternal and Child Health Surveillance of China, West China Second University Hospital, Sichuan University, Chengdu, Sichuan, China

\section{Peiran Chen}

National Office for Maternal and Child Health Surveillance of China, West China Second University Hospital, Sichuan University, Chengdu, Sichuan, China

\section{Yanxia Xie}

National Office for Maternal and Child Health Surveillance of China, West China Second University Hospital, Sichuan University, Chengdu, Sichuan, China

\section{Juan Liang}

National Office for Maternal and Child Health Surveillance of China, West China Second University Hospital, Sichuan University, Chengdu, Sichuan, China

\section{Jun Zhu ( $\nabla$ zhujun028@163.com )}

National Office for Maternal and Child Health Surveillance of China, West China Second University Hospital, Sichuan University, Chengdu, Sichuan, China

\section{Research Article}

Keywords: Pregnancy complications, Uterine rupture, Risk factors, Large for gestational age, Preterm delivery

Posted Date: October 8th, 2021

DOI: https://doi.org/10.21203/rs.3.rs-424294/v2

License: (c) (i) This work is licensed under a Creative Commons Attribution 4.0 International License. Read Full License 
Version of Record: A version of this preprint was published at BMC Pregnancy and Childbirth on February 16th, 2022. See the published version at https://doi.org/10.1186/s12884-022-04465-w. 


\section{Abstract}

Background: The goal of this study was to investigate whether pregnancy complications are associated with an increased risk of uterine rupture (UR) and how that risk changes with gestational age.

Methods: We obtained all data from China's National Maternal Near Miss Surveillance System (NMNMSS) between 2012 and 2018. Poisson regression analysis was used to assess the risk of UR with pregnancy complications (preeclampsia, gestational diabetes mellitus, placental abruption, placenta previa and placenta percreta) among 9,454,239 pregnant women. Furthermore, we analysed the risks of UR with pregnancy complications in different gestational age groups.

Results: Compared with women without pregnancy complications, those with different complications (except for preeclampsia) had a 1- to 3-fold greater risk of UR. These associations also persisted in women without a previous caesarean delivery. Moreover, an increased risk of UR before term birth was observed among women with gestational diabetes mellitus, placental abruption and placenta percreta. A large for gestational age (LGA) foetus increased the risk of UR in women with gestational diabetes mellitus, especially at 32 to 36 weeks gestation.

Conclusions: Better quality antenatal care and early intervention for women with these pregnancy complications are needed to minimize the incidence of UR.

\section{Background}

Uterine rupture (UR) is a tear in the uterine wall that occurs before or during labour. It poses considerable risks for adverse maternal and perinatal outcomes, including serious health risks for both mother (e.g., maternal death) and foetus (e.g., stillbirth, neonatal death)[1, 2]. Mounting evidence supports that a history of caesarean section is a major risk factor for UR in subsequent pregnancies[1, 3], and the risk increases with the number of previous cesarean deliveries [4, 5]. The incidence of UR varies across countries, ranging from 0.18 to 9 cases per 1,000 pregnant women[1, 6, 7]. Many countries have created policies to decrease caesarean rates[8,9], but the UR rate has increased over the years[10,11]. It is unknown whether the increasing rate of UR is due to the effects of potential risk factors related to a history of caesarean section.

Evidence suggests that women who have a history of caesarean section may be at increased risk of pregnancy complications, such as placental abruption, placenta previa, placenta percreta, gestational diabetes mellitus and preeclampsia[12-14]. These pregnancy complications may be partly considered manifestations of dysfunctional placental function[15, 16]. To date, placenta percreta has been reported to be associated with an increased risk of UR[17, 18]. However, there are limited data on the association of other pregnancy complications with UR. A few studies with small sample sizes have analysed the impacts of diabetes and hypertension on the risk of UR[7, 19,20], but these studies have yielded different conclusions. In addition, current guidelines only discuss the impact of vaginal trial delivery after caesarean section on the occurrence of UR [21,22], but the effects of pregnancy complications related to 
previous caesarean deliveries have not been reported. If a link between pregnancy complications and UR is confirmed, it may provide additional preventive measures.

We hypothesize that pregnancy complications (preeclampsia, gestational diabetes mellitus, placental abruption, placenta previa, placenta percreta) may be associated with an increased risk of UR. Therefore, our study analysed more than 9 million singleton pregnant women from China's National Maternal Near Miss Surveillance System (NMNMSS) to evaluate whether these pregnancy complications were associated with the risk of UR. By analysing the impact of each pregnancy complication in different gestational age groups, we provided insights into the early interventions that would contribute to reducing the incidence of UR in pregnant women.

\section{Methods}

\section{Design and setting}

We obtained data from China's NMNMSS between 2012 and 2018. The system covers 438 hospitals in 326 districts or counties throughout 30 provinces, each of which manages more than 1,000 deliveries annually[11]. Data collected included sociodemographic characteristics, obstetric history, pregnancy complications, and pregnancy outcomes of all pregnant or postpartum women in a hospital. Doctors in each hospital were trained to collect data prospectively from admission to discharge. Quality assurance was ensured by staff from county-level, municipal-level and provincial-level maternal and child health hospitals 1-2 times a year. At the same time, the National Office for Maternal and Child Health Surveillance verified the quality of the records by selecting 6-8 hospitals randomly in each province once a year[23].

\section{Study population}

We restricted the analysis to pregnant women with singleton births who delivered at or after 28 complete weeks of gestation. Women with multiple pregnancies were not included because they are prone to pregnancy complications[24] and UR[25]. Women lacking information on delivery method, history of caesarean section, or gravidity were excluded. We also excluded women with an unlikely combination of gravidity and parity. This left a total of 9,454,239 women for the study.

\section{Variable definition}

UR was defined as uterine or lower uterine dehiscence during late pregnancy or delivery[26]. According to the degree of dehiscence, UR can be divided into complete UR (tearing in all layers of the uterine wall) and incomplete UR (tearing in the muscular layers)[10]. Common clinical manifestations of UR include foetal distress, sudden tearing uterine pain, cessation of uterine contractions and abnormal vaginal bleeding[27]. UR was diagnosed by a health professional with imaging techniques (magnetic resonance imaging or ultrasound examination)[28]; or during emergency caesarean delivery; or peripartum hysterectomy or laparotomy after vaginal birth[3]. Unfortunately, UR is captured as a dichotomous 
variable (yes/no) in the NMNMSS, and the type of rupture is lacking. We identified five pregnancy complications related to previous caesarean delivery for analysis: preeclampsia, gestational diabetes mellitus, placental abruption, placenta previa and placenta percreta[13,14]. Preeclampsia included pregnancies with preeclampsia, eclampsia or HELLP (haemolysis, elevated liver enzymes and low platelets) syndrome, as well as chronic hypertension with superimposed preeclampsia. Gestational diabetes was diagnosed by an oral glucose tolerance test (OGTT) during pregnancy. Placental abruption was defined as the premature separation of the implanted placenta before delivery. Placenta previa was defined as the placenta covering the internal os of the cervix. Placenta percreta, as the most severe grade of the placenta accreta spectrum disorders, occurs when the chorionic villi penetrate the uterine serosa[29].

We selected variables that may be related to the occurrence of UR, including region, hospital level, education level (none, primary school, middle school, high school, college or higher), maternal age at delivery $(<20,20-24,25-29,30-34,35-39$ and $\geq 40$ years), the number of antenatal visits (none, $1-3$, $4-6,7-9, \geq 10)$, gravidity $(1,2-3, \geq 4)$, parity $(0,1, \geq 2)$, number of previous caesarean deliveries $(0,1$, $\geq 2$ ), foetal presentation (cephalic and other abnormal lies), gestational age, birthweight, and mode of delivery (vaginal delivery and caesarean section). We divided China's regions into three categories (eastern, central and western) and classified hospitals into three levels (the first level represents the smallest hospital) according to standard definitions[23]. Gestational age was defined based on ultrasound measurement results or estimated from the date of the last menstrual period and classified as early preterm (28-33 weeks), late preterm (34-36 weeks), or term ( $\geq 37$ weeks). Large for gestational age (LGA) was defined as a gestational age-adjusted birth weight above the 90th percentile[30]. Other factors thought to be associated with UR included gestational hypertension, chronic hypertension, heart disease, hepatic disease, severe anaemia (haemoglobin concentration lower than $70 \mathrm{~g} / \mathrm{L}$ ), infection (excluding abortion-related infection, puerperal infection and abdominal incision infection), thrombophlebitis, renal disease, lung disease, and connective tissue disorders.

\section{Statistical Analysis}

\section{Primary analysis}

We expressed the UR rate as the number of pregnant women with UR per 1,000 pregnant women. Since some women giving birth in township hospitals were not included in the NMNMSS, we weighted the UR rate for the sampling distribution of the population according to the 2010 census of China, as detailed elsewhere[23]. Moreover, a history of caesarean section is a major risk factor for UR in subsequent pregnancies[1, 3], and the risk increases with the number of previous caesarean deliveries[4, 5]. Thus, we calculated the previous caesarean deliveries adjusted rate of UR in women by using the margins command in Stata[31].

We classified pregnancy complications into mutually exclusive categories: preeclampsia, gestational diabetes mellitus, placental abruption, placenta previa, and placenta percreta. We used Poisson regression with a robust variance estimator to assess the association of UR with pregnancy 
complications, reporting the results from two models. The reference for each model was women without any of the five pregnancy complications. Model 1 describes the adjusted relative risk (aRR) and 95\% confidence Interval $(\mathrm{Cl})$, taking into account the sampling distribution of the population and birth clustering within hospitals, medical institutions, and pregnant women's sociodemographic and clinical factors that might contribute to the observed associations. Model 2 adjusted for the covariates in Model 1 as well as the number of previous caesarean deliveries $(0,1, \geq 2)$ and LGA (yes/no). We did not adjust for gestational age or final mode of delivery because they included consequences of UR (i.e., laparotomy due to UR). To identify the most robust and stable model, we investigated both multicollinearity and model goodness-of-fit.

Due to a history of caesarean section related to both pregnancy complications[1, 3] and UR[12-14], pregnancy complications may be only an intermediate factor in the causal chain between a history of caesarean section and the risk of UR. We repeated the association analysis of pregnancy complications with UR only in women without previous caesarean delivery.

\section{Secondary analysis}

\section{Restricting to a group of women}

To investigate the association between pregnancy complications and UR without potential maternal confounding factors (advanced maternal age[1] and multiple gravidities[32]), we performed sensitivity analyses excluding women with advanced maternal age ( $\geq 35$ years) and/or multiple gravidities $(\geq 4)$. Given the possible impacts of abnormal foetal presentation and macrosomia on the occurrence of UR[33], we restricted the association analysis to women with offspring having a cephalic lie and a birth weight of less than or equal to $4000 \mathrm{~g}$.

\section{Co-occurrence of pregnancy complications and UR risk}

Pregnancy complications may co-occur in a given pregnancy. We therefore repeated model 1 and 2 testing for the associations between having at least two or more pregnancy complications and the risk of UR. Because the numbers were too small to assess unique combinations of pregnancy complications, we modelled the variables "no pregnancy complications", "any one pregnancy complication", and "any two or more pregnancy complications" in a single model.

\section{Risk of UR in different gestational age groups}

To explore the risk of UR with pregnancy complications in different gestational age groups, we compared the UR rates in women for each pregnancy complication and gestational age against those in women without pregnancy complications at 28-33 weeks of gestational age, using model 1 and 2 .

\section{Role of large for gestational age}


Because LGA is associated with gestational diabetes mellitus[34], we repeated model 1 and 2 testing to analyse a possible effect of LGA foetuses on the risk of UR among women with gestational diabetes mellitus.

\section{Trends over time in UR rates}

To examine trends over time in UR rates among women with pregnancy complications, we repeated model 1 and 2 by including the year of study period as a continuous variable.

Statistical analysis was performed using Stata (version 16.0, Stata Corp LP., College Station, United States of America). $\mathrm{P}<0.05$ (2-sided) was considered statistically significant.

\section{Patient involvement}

Informed consent from the patients was waived by the Ethics Committee, as the data used in our study were obtained from a national routine surveillance system established by the government. Data use was authorized by the National Health Commission, and data provided to us were deidentified.

\section{Results}

Of the 9,454,239 pregnant women enrolled in this study, 885,087 (9.4\%) women had pregnancy complications. Compared with women without pregnancy complications, women with pregnancy complications tended to be older, to have multiple gravidities and to have had previous caesarean deliveries. At the time of birth, women with pregnancy complications had a higher percentage of abnormal foetal presentations and LGA. Details are summarized in Table 1. 
Table 1

Maternal and fetal characteristics of 9,454,239 pregnant women with singleton births

\section{Sociodemographic characteristic}

Women without pregnancy complications, $\mathrm{n}(\%)$

$n=8,569,152(90.6 \%)$
Women with pregnancy complications, $\mathrm{n}(\%)$

$n=885,087(9.4 \%)$

\section{Region of China}

$\begin{array}{llll}\text { East } & 2,407,580(88.5) & 313,912(11.5) & 0<0 \\ \text { Central } & 3,464,042(91.9) & 304,138(8.1) & \\ \text { West } & 2,697,530(91.0) & 267,037(9.0) & 0.001 \\ \text { Hospital level } & & & \\ \text { Unknown } & 457,421(95.2) & 23,264(4.8) & \\ \text { Level 1 } & 569,527(96.8) & 18,536(3.2) & \\ \text { Level 2 } & 4,094,405(93.3) & 295,839(6.7) & \end{array}$

\section{Maternal education}

\begin{tabular}{llll} 
None & $42,037(90.3)$ & $4,523(9.7)$ & $0<0$ \\
Primary school & $259,703(91.8)$ & $23,323(8.2)$ & \\
Middle school & $2,821,827(93.9)$ & $182,270(6.1)$ \\
\hline High school & $2,309,079(90.8)$ & $235,360(9.2)$ \\
\hline College or higher & $2,977,190(87.9)$ & $409,826(12.1)$ \\
\hline Unknown & $159,316(84.2)$ & $29,785(15.8)$ \\
\hline Mother's age, years & & \\
\hline$<20$ & $229,174(96.5)$ & $8,387(3.5)$ & 0.001 \\
\hline $20-24$ & $1,742,770(95.3)$ & $86,051(4.7)$ \\
\hline $25-29$ & $3,554,091(92.1)$ & $303,454(7.9)$ \\
\hline $30-34$ & $1,941,052(87.7)$ & $272,915(12.3)$ \\
\hline $35-39$ & $699,991(82.3)$ & $150,716(17.7)$ \\
\hline$\geq 40$ & $153,702(77.4)$ & $45,001(22.6)$ \\
\hline Unknown & $248,372(93.0)$ & $18,563(7.0)$ \\
\hline Antenatal care visits & & \\
\hline
\end{tabular}




\begin{tabular}{|c|c|c|c|}
\hline \multirow[t]{2}{*}{$\begin{array}{l}\text { Sociodemographic } \\
\text { characteristic }\end{array}$} & $\begin{array}{l}\text { Women without pregnancy } \\
\text { complications, } n(\%)\end{array}$ & $\begin{array}{l}\text { Women with pregnancy } \\
\text { complications, } \mathrm{n}(\%)\end{array}$ & \multirow[t]{2}{*}{$\begin{array}{l}P \\
\text { value }\end{array}$} \\
\hline & $n=8,569,152(90.6 \%)$ & $\mathrm{n}=885,087(9.4 \%)$ & \\
\hline None & $106,694(92.0)$ & $9,327(8.0)$ & \multirow{6}{*}{$\begin{array}{l}P< \\
0.001\end{array}$} \\
\hline $1-3$ & $586,349(93.0)$ & $43,983(7.0)$ & \\
\hline $4-6$ & $2,599,192(93.9)$ & $169,815(6.1)$ & \\
\hline $7-9$ & $2,576,046(90.5)$ & $271,105(9.5)$ & \\
\hline$\geq 10$ & $2,480,962(87.5)$ & $353,423(12.5)$ & \\
\hline Unknown & $219,909(85.5)$ & $37,434(14.5)$ & \\
\hline \multicolumn{4}{|l|}{ Gravidity } \\
\hline 1 & $3,440,750(92.0)$ & $297,971(8.0)$ & \multirow{3}{*}{$\begin{array}{l}P< \\
0.001\end{array}$} \\
\hline $2-3$ & $4,092,602(90.7)$ & $421,269(9.3)$ & \\
\hline$\geq 4$ & $1,035,800(86.2)$ & $165,847(13.8)$ & \\
\hline \multicolumn{4}{|l|}{ Parity } \\
\hline 0 & $4,848,051(91.1)$ & $474,646(8.9)$ & \multirow{3}{*}{$\begin{array}{l}P< \\
0.001\end{array}$} \\
\hline 1 & $3,209,056(90.0)$ & $356,733(10.0)$ & \\
\hline$\geq 2$ & $512,045(90.5)$ & $53,708(9.5)$ & \\
\hline \multicolumn{4}{|c|}{ Previous caesarean deliveries } \\
\hline 0 & $7,274,553(91.3)$ & $692,669(8.7)$ & \multirow{3}{*}{$\begin{array}{l}P< \\
0.001\end{array}$} \\
\hline 1 & $1,220,781(87.1)$ & $180,214(12.9)$ & \\
\hline$\geq 2$ & $73,818(85.8)$ & $12,204(14.2)$ & \\
\hline \multicolumn{4}{|l|}{ Foetal presentation } \\
\hline Cephalic & $8,298,133(90.8)$ & $839,684(9.2)$ & \multirow{3}{*}{$\begin{array}{l}P< \\
0.001\end{array}$} \\
\hline abnormal lies & $267,253(85.8)$ & $44,191(14.2)$ & \\
\hline Unknown & $3,766(75.7)$ & $1,212(24.3)$ & \\
\hline \multicolumn{4}{|c|}{ Large for gestational age } \\
\hline No & $7,759,647(91.0)$ & $764,956(9.0)$ & \multirow{2}{*}{$\begin{array}{l}P< \\
0.001\end{array}$} \\
\hline Yes & $809,505(87.1)$ & $120,131(12.9)$ & \\
\hline
\end{tabular}




\begin{tabular}{|c|c|c|c|}
\hline \multirow[t]{2}{*}{$\begin{array}{l}\text { Sociodemographic } \\
\text { characteristic }\end{array}$} & $\begin{array}{l}\text { Women without pregnancy } \\
\text { complications, } \mathrm{n}(\%)\end{array}$ & $\begin{array}{l}\text { Women with pregnancy } \\
\text { complications, } \mathrm{n}(\%)\end{array}$ & \multirow[t]{2}{*}{$\begin{array}{l}P \\
\text { value }\end{array}$} \\
\hline & $\mathrm{n}=8,569,152(90.6 \%)$ & $n=885,087(9.4 \%)$ & \\
\hline Vaginal & $4,944,933(93.9)$ & $321,071(6.1)$ & \multirow{2}{*}{$\begin{array}{l}P< \\
0.001\end{array}$} \\
\hline Caesarean section & $3,624,219(86.5)$ & $564,016(13.5)$ & \\
\hline
\end{tabular}

Overall, $8.8 \%$ of the women had a single pregnancy complication $(830,648)$ and $0.6 \%$ had two or more $(54,439)$. Thus, most pregnancy complications occurred as single events (Fig. 1). Among these, gestational diabetes mellitus was the most common pregnancy complication, followed by preeclampsia, placenta previa, placental abruption and placenta percreta.

\section{Trends over time in UR rates}

There were 16,949 pregnant women with UR, giving a weighted UR rate of 1.6 cases per thousand pregnant women. Figure 2 shows that the rate of UR was markedly higher in women with pregnancy complications than in women without pregnancy complications, irrespective of medical institution or the pregnant woman's sociodemographic and clinical factors. Moreover, the rate of UR in women with pregnancy complications increased as the ratio of women with pregnancy complications increased between 2012 and 2018. However, there was no change in the UR rate in women with pregnancy complications over time after adjustment for the number of previous caesarean deliveries and all other risk factors (Model 2, aRR: 1.44, 95\% Cl: 0.91-2.29, Additional file Table S1). Similarly, the UR rate did not change over time in women with each pregnancy complication after adjustment (Additional file Table S1).

\section{Risk of UR stratified by history of caesarean section}

The incidence of UR varied in women with different single-complications (Table 2), with the highest rate in women with placenta percreta. Except for preeclampsia, the other four pregnancy complications were associated with a significantly increased risk of UR after adjustment for risk factors (Table 2). After excluding women with previous caesarean deliveries, these associations were substantially elevated. Among women without previous caesarean delivery, the adjusted risk of UR was 1.41 (95\% Cl: 1.21-1.65) for women with gestational diabetes mellitus, 5.03 (95\% Cl: 3.40-7.42) for women with placental abruption, 5.38 (95\% Cl: 3.76-7.70) for women with placenta previa, and 12.79 (95\% Cl: 7.69-21.27) for women with placenta percreta (Table 2). 
Table 2

Risk of uterine rupture with pregnancy complications

\begin{tabular}{|c|c|c|c|c|c|c|c|}
\hline $\begin{array}{l}\text { Pregnancy } \\
\text { complications * }\end{array}$ & No. UR & $\begin{array}{l}\text { UR } \\
\text { rate }^{\#}\end{array}$ & $\begin{array}{l}\text { Adjusted } \\
\text { UR rate } \# \#\end{array}$ & $\begin{array}{l}\text { aRR }(95 \% \mathrm{Cl}) \text { : } \\
\text { Model } 1^{\mathrm{a}}\end{array}$ & $\begin{array}{l}P \\
\text { value }\end{array}$ & $\begin{array}{l}\text { aRR } \\
(95 \% \mathrm{Cl}): \\
\text { Model } 2^{\mathrm{b}}\end{array}$ & $\begin{array}{l}P \\
\text { value }\end{array}$ \\
\hline \multicolumn{8}{|l|}{ All women } \\
\hline None & 13,651 & 1.4 & 1.4 & 1 (reference) & & $\begin{array}{l}1 \\
\text { (reference) }\end{array}$ & \\
\hline Preeclampsia & 338 & 1.7 & 1.5 & $\begin{array}{l}0.97(0.76- \\
1.24)\end{array}$ & 0.825 & $\begin{array}{l}0.89(0.70- \\
1.14)\end{array}$ & 0.358 \\
\hline $\begin{array}{l}\text { Gestational } \\
\text { diabetes mellitus }\end{array}$ & 1,812 & 3.2 & 2.5 & $\begin{array}{l}1.36(1.16- \\
1.60)\end{array}$ & $\dot{<} 0.001$ & $\begin{array}{l}1.20(1.03- \\
1.41)\end{array}$ & 0.020 \\
\hline $\begin{array}{l}\text { Placental } \\
\text { abruption }\end{array}$ & 175 & 5.6 & 5.4 & $\begin{array}{l}2.44(1.79- \\
3.33)\end{array}$ & $<.001$ & $\begin{array}{l}2.74(2.04- \\
3.66)\end{array}$ & $<0.001$ \\
\hline Placenta previa & 520 & 6.4 & 3.7 & $\begin{array}{l}2.13(1.64- \\
2.77)\end{array}$ & $<.001$ & $\begin{array}{l}1.72(1.33- \\
2.22)\end{array}$ & $<.001$ \\
\hline Placenta percreta & 88 & 13.5 & 6.2 & $\begin{array}{l}3.81(2.48- \\
5.83)\end{array}$ & $\dot{0} 001$ & $\begin{array}{l}2.64(1.71- \\
4.07)\end{array}$ & $<.001$ \\
\hline \multicolumn{8}{|c|}{ Women without previous caesarean delivery } \\
\hline None & 1,808 & 0.2 & - & 1 (reference) & & $\begin{array}{l}1 \\
\text { (reference) }\end{array}$ & \\
\hline Preeclampsia & 46 & 0.3 & - & $\begin{array}{l}1.02(0.69- \\
1.49)\end{array}$ & 0.938 & $\begin{array}{l}1.02(0.69- \\
1.49)\end{array}$ & 0.933 \\
\hline $\begin{array}{l}\text { Gestational } \\
\text { diabetes mellitus }\end{array}$ & 199 & 0.4 & - & $\begin{array}{l}1.43(1.22- \\
1.67)\end{array}$ & $\stackrel{<}{0.001}$ & $\begin{array}{l}1.41(1.21- \\
1.65)\end{array}$ & $<0.001$ \\
\hline $\begin{array}{l}\text { Placental } \\
\text { abruption }\end{array}$ & 46 & 1.9 & - & $\begin{array}{l}4.98(3.38- \\
7.34)\end{array}$ & $<.001$ & $\begin{array}{l}5.03(3.40- \\
7.42)\end{array}$ & $\begin{array}{l}<.001 \\
0.001\end{array}$ \\
\hline Placenta previa & 116 & 2.1 & - & $\begin{array}{l}5.36(3.76- \\
7.66)\end{array}$ & $\dot{0} 001$ & $\begin{array}{l}5.38(3.76- \\
7.70)\end{array}$ & $<.001$ \\
\hline Placenta percreta & 25 & 6.7 & - & $\begin{array}{l}12.77(7.69- \\
21.23)\end{array}$ & $<.001$ & $\begin{array}{l}12.79 \\
(7.69- \\
21.27)\end{array}$ & $<0.001$ \\
\hline \multicolumn{8}{|c|}{$\begin{array}{l}\text { *Each one of pregnancy complications has no other complications. None: Women with none of the } \\
\text { five pregnancy complications. }\end{array}$} \\
\hline \multicolumn{8}{|c|}{ \# Weighted uterine rupture rate per 1000 women } \\
\hline
\end{tabular}




\begin{tabular}{|llllllll|}
\hline $\begin{array}{l}\text { Pregnancy } \\
\text { complications }^{*}\end{array}$ & No. UR & $\begin{array}{l}\text { UR } \\
\text { rate }^{\#}\end{array}$ & $\begin{array}{l}\text { Adjusted } \\
\text { UR rate }^{\#}\end{array}$ & $\begin{array}{l}\text { aRR }(95 \% \mathrm{Cl}): \\
\text { Model 1 }\end{array}$ & $\begin{array}{l}P \\
\text { value }\end{array}$ & $\begin{array}{l}\text { aRR } \\
(95 \% \mathrm{Cl}):\end{array}$ & $\begin{array}{l}P \\
\text { Model } 2^{\mathrm{b}}\end{array}$
\end{tabular}

a Model 1: adjusted for sampling distribution of population and clustering of births within hospitals, region, hospital level, the number of antenatal visits, the women's educational level, maternal age at delivery, parity, foetal presentation, gestational hypertension, chronic hypertension, heart disease, hepatic disease, severe anaemia, infection, thrombophlebitis, renal disease, lung disease, connective tissue disorders.

b Model 2: adjusted for Model 1 as well as the number of previous caesarean deliveries $(0,1, \geq 2)$ and large for gestational age (yes/no).

The results were similar after restricting the dataset to those women without advanced maternal age and multiple gravidities (Additional file Table S2). When women with offspring having abnormal foetal presentation and a birth weight of more than $4000 \mathrm{~g}$ were excluded, the risks of UR with pregnancy complications were largely unchanged (Additional file Table S3).

\section{Co-occurrence of pregnancy complications and UR risk}

Compared with having none of the five pregnancy complications, having two or more complications was associated with a statistically significant almost 1.42-fold risk of UR (Model 1, aRR: $1.88,95 \%$ Cl: $1.51-$ 2.34; Model 2, aRR: 1.42, 95\% Cl:1.14-1.77).

\section{Risk of UR in different gestational age groups}

For a small proportion $(14.9 \%, 2,531$ of 16,949$)$ of women with UR, the rupture occurred before term birth. An increased risk of UR before term birth was observed among women with gestational diabetes mellitus, placental abruption and placenta percreta (Table 3). Women with placenta percreta had the highest risk of UR at 28 to 33 weeks gestation (Model 2, aRR: 6.21, 95\% Cl: 3.43-11.24). The risk of UR among women with gestational diabetes mellitus was only observed at 34 to 36 weeks gestation (Model 2, aRR: $1.43,95 \% \mathrm{Cl}: 1.03-1.97)$. Moreover, the risk of UR among women with placenta previa was only observed at term (Model 2, aRR: 1.41, 95\% Cl: 1.08-1.86). 
Table 3

Association between pregnancy complications and uterine rupture by gestational age group

\section{Pregnancy complications * Gestational age group (week)}

Early preterm (28-33) Late preterm (34-36) Term ( $\geq 37)$

\section{No. UR}

None

Preeclampsia

Gestational diabetes mellitus

Placental abruption

Placenta previa

Placenta percreta

UR rate (Adjusted UR rate $^{\# \#}$ )

None

Preeclampsia

Gestational diabetes mellitus

Placental abruption

Placenta previa

Placenta percreta

aRR (95\%Cl): Model $1^{\mathrm{a}}$

None

1 (reference)

$1.72(1.29-2.30)^{c}$

$0.78(0.44-1.38)$

$0.25(0.14-0.44)^{c}$

$1.29(0.96-1.73)$

$1.69(1.17-2.46)^{d}$

$1.27(0.89-1.82)$

$7.99(4.43-14.40)^{c}$
1,430

72

198

51

138

11

$3.8(3.4)$

$2.3(1.7)$

$6.4(4.3)$

$7.5(5.7)$

$5.9(3.0)$

$15.7(6.0)$
11,909

246

1,573

84

336

61

\begin{tabular}{|c|c|c|c|}
\hline Preeclampsia & $0.25(0.14-0.44)^{c}$ & $0.78(0.44-1.38)$ & $0.89(0.61-1.31)$ \\
\hline Gestational diabetes mellitus & $1.29(0.96-1.73)$ & $1.84(1.32-2.57)^{c}$ & $1.00(0.75-1.33)$ \\
\hline Placental abruption & $1.69(1.17-2.46)^{d}$ & $2.23(1.62-3.06)^{c}$ & $1.85(1.38-2.50)^{c}$ \\
\hline Placenta previa & $1.27(0.89-1.82)$ & $1.34(0.89-2.03)$ & $1.91(1.44-2.53)^{c}$ \\
\hline Placenta percreta & $7.99(4.43-14.40)^{c}$ & $3.10(1.65-5.82)^{c}$ & $2.52(1.73-3.69)^{c}$ \\
\hline \multicolumn{4}{|l|}{ aRR (95\%Cl): Model $2^{b}$} \\
\hline None & 1 (reference) & $1.47(1.12-1.92)^{d}$ & $0.67(0.53-0.85)^{d}$ \\
\hline Preeclampsia & $0.21(0.12-0.38)^{c}$ & $0.63(0.36-1.12)$ & $0.77(0.51-1.14)$ \\
\hline
\end{tabular}




\begin{tabular}{|c|c|c|c|}
\hline \multirow[t]{2}{*}{ Pregnancy complications * } & \multicolumn{3}{|c|}{ Gestational age group (week) } \\
\hline & Early preterm (28-33) & Late preterm (34-36) & Term ( $\geq 37)$ \\
\hline Gestational diabetes mellitus & $1.13(0.84-1.53)$ & $1.43(1.03-1.97)^{e}$ & $0.82(0.62-1.08)$ \\
\hline Placental abruption & $1.60(1.00-2.31)^{e}$ & $2.13(1.55-2.92)^{c}$ & $2.12(1.60-2.81)^{c}$ \\
\hline Placenta previa & $1.08(0.76-1.54)$ & $0.97(0.67-1.43)$ & $1.41(1.08-1.86)^{e}$ \\
\hline Placenta percreta & $6.21(3.43-11.24)^{c}$ & $1.92(0.99-3.71)$ & $1.60(1.08-2.38)^{\mathrm{e}}$ \\
\hline \multicolumn{4}{|c|}{$\begin{array}{l}\text { *Each one of pregnancy complications has no other complications. None: Women with none of the } \\
\text { five pregnancy complications. }\end{array}$} \\
\hline \multicolumn{4}{|c|}{ \# Weighted uterine rupture rate per 1000 women } \\
\hline \multicolumn{4}{|c|}{ \#\# Weighted, and previous caesarean deliveries adjusted uterine rupture rate per 1000 women } \\
\hline \multicolumn{4}{|c|}{$\begin{array}{l}\text { a Model 1: adjusted for sampling distribution of population and clustering of births within hospitals, } \\
\text { region, hospital level, the number of antenatal visits, the women's educational level, maternal age at } \\
\text { delivery, parity, foetal presentation, gestational hypertension, chronic hypertension, heart disease, } \\
\text { hepatic disease, severe anaemia, infection, thrombophlebitis, renal disease, lung disease, connective } \\
\text { tissue disorders. }\end{array}$} \\
\hline \multicolumn{4}{|c|}{$\begin{array}{l}\text { b Model 2: adjusted for Model } 1 \text { as well as the number of previous caesarean deliveries }(0,1, \geq 2) \text { and } \\
\text { large for gestational age (yes/no). }\end{array}$} \\
\hline${ }^{\mathrm{c}} P<0.001 ; \mathrm{d} P<0.01 ;$ e $P<0.0$ & & & \\
\hline
\end{tabular}

\section{Role of large for gestational age}

The risk of UR in women with gestational diabetes mellitus without an LGA foetus was 1.18-fold (Model 2, aRR: $1.18,95 \% \mathrm{Cl}: 1.00-1.38)$, and the risk was slightly larger in women with gestational diabetes mellitus and an LGA foetus (Model 2, aRR: 1.28, 95\% Cl: 1.09-1.50) (Table 4). Among women at 34-36 weeks gestational age, the association between gestational diabetes mellitus with an LGA foetus and UR was slightly elevated (Model 2, aRR: 1.40, 95\% Cl: 1.09-1.79) (Table 4). 
Table 4

The association between gestational diabetes mellitus and uterine rupture

$\begin{array}{llllllll}\begin{array}{l}\text { Gestational diabetes } \\ \text { mellitus }\end{array} & \text { No. UR } & \begin{array}{l}\text { UR } \\ \text { rate }^{\#}\end{array} & \begin{array}{l}\text { Adjusted } \\ \text { UR rate }^{\# \#}\end{array} & \begin{array}{l}\text { aRR } \\ (95 \% \mathrm{Cl}): \\ \text { Model 1 }\end{array} & \begin{array}{l}P \\ \text { value }\end{array} & \begin{array}{l}\text { aRR } \\ (95 \% \mathrm{Cl}):\end{array} & \begin{array}{l}P \\ \text { model 2 }\end{array}\end{array}$

\section{All women}

None only

$12,138 \quad 1.4 \quad 1.5$

1

(reference)

1

(reference)

None and LGA

$\begin{array}{lll}1,513 & 1.7 & 1.3\end{array}$

$1.02(0.95-$

0.550

0.96

1.11)

$(0.89-$

0.335

$1.04)$

\begin{tabular}{|c|c|c|c|c|c|c|}
\hline $\begin{array}{l}\text { Gestational diabetes } \\
\text { mellitus only }\end{array}$ & 1,452 & 3.0 & 2.5 & $\begin{array}{l}1.32(1.11- \\
1.56)\end{array}$ & 0.001 & $\begin{array}{l}1.18 \\
(1.00- \\
1.38)\end{array}$ \\
\hline $\begin{array}{l}\text { Gestational diabetes } \\
\text { mellitus and LGA }\end{array}$ & 360 & 4.1 & 2.5 & $\begin{array}{l}1.59(1.34- \\
1.88)\end{array}$ & $\begin{array}{l}< \\
0.001\end{array}$ & $\begin{array}{l}1.28 \\
(1.09- \\
1.50)\end{array}$ \\
\hline
\end{tabular}

Women at 34-36 weeks gestational age

\begin{tabular}{|c|c|c|c|c|c|c|c|}
\hline None only & 1,274 & 3.7 & 4.3 & $\begin{array}{l}1 \\
\text { (reference) }\end{array}$ & & $\begin{array}{l}1 \\
\text { (referen }\end{array}$ & \\
\hline None and LGA & 156 & 4.2 & 3.6 & $\begin{array}{l}1.02(0.81- \\
1.30)\end{array}$ & 0.841 & $\begin{array}{l}0.92 \\
(0.73- \\
1.15)\end{array}$ & 0.459 \\
\hline $\begin{array}{l}\text { Gestational diabetes } \\
\text { mellitus only }\end{array}$ & 140 & 5.4 & 4.8 & $\begin{array}{l}1.00(0.81- \\
1.23)\end{array}$ & 0.968 & $\begin{array}{l}0.92 \\
(0.76- \\
1.12)\end{array}$ & 0.414 \\
\hline $\begin{array}{l}\text { Gestational diabetes } \\
\text { mellitus and LGA }\end{array}$ & 58 & 10.9 & 6.5 & $\begin{array}{l}1.77(1.38- \\
2.26)\end{array}$ & ${ }_{0.001}^{<}$ & $\begin{array}{l}1.40 \\
(1.09- \\
1.79)\end{array}$ & 0.009 \\
\hline
\end{tabular}

* Gestational diabetes mellitus: Women with no other four complications. None: Women with none of the five pregnancy complications. LGA: Large for gestational age.

\# Weighted uterine rupture rate per 1000 women

\#\# Weighted, and previous caesarean deliveries adjusted uterine rupture rate per 1000 women

a Model 1: adjusted for sampling distribution of population and clustering of births within hospitals, region, hospital level, the number of antenatal visits, the women's educational level, maternal age at delivery, parity, foetal presentation, gestational hypertension, chronic hypertension, heart disease, hepatic disease, severe anaemia, infection, thrombophlebitis, renal disease, lung disease, connective tissue disorders.

${ }^{b}$ Model 2: adjusted for Model 1 as well as the number of previous caesarean deliveries $(0,1, \geq 2)$. 


\section{Discussion}

Using data from more than 9 million singleton pregnant women in China, we found that approximately one-tenth of all women had pregnancy complications, and most occurred as single events. The incidence of UR varied in women with different single-complications, and the highest rate was observed in women with placenta percreta. Gestational diabetes mellitus, placental abruption, placenta previa and placenta percreta were associated with a substantially increased risk of UR, andthe risks for UR were 1- to 3-fold higher among women with these pregnancy complications. These associations persisted in women without previous caesarean delivery. Moreover, a significantly increased risk of UR before term birth was observed in women with gestational diabetes mellitus, placental abruption and placenta percreta. An LGA foetus increased the risk of UR in women with gestational diabetes mellitus, especially at 32 to 36 weeks gestation.

In our data, the largest increased risk of UR was seen for placenta percreta (aRR: 2.64, 95\% Cl: 1.714.07). Among women without previous caesarean delivery, the risk of UR was approximately 13 times higher in women with placenta percreta than in those without pregnancy complications (aRR: 12.79, 95\% $\mathrm{Cl}$ : 7.69-21.27). A large retrospective cohort study from Negev found that pregnant women with placenta accreta spectrum disorders (including placenta percreta) had a significantly increased risk of UR (OR: 6.42, 95\% Cl: 2.0-20.4)[17]. Moreover, previous research found that UR occurs in women without a history of caesarean section[3,35,36] and identified spontaneous UR due to placenta percreta as occurring in a primigravida woman without prior uterine operation[37]. Thus, our findings are consistent with previous studies, suggesting that placenta percreta (especially without previous caesarean delivery) may increase the risk of UR.

To date, few studies have reported the impact of other pregnancy complications on the occurrence of UR and have mainly focused on the impact of preeclampsia/eclampsia and diabetes mellitus. However, there are inconsistent conclusions regarding the effects of preeclampsia/eclampsia and gestational diabetes mellitus on UR risk. A population-based Negev study of 138 pregnant women with UR found that hypertension disorders (including preeclampsia/eclampsia) were associated with a twofold increased risk of UR (OR: 2.05, 95\% Cl: 1.20-3.50), but diabetes mellitus (prepregnancy and gestation) was not related to the risk of UR (OR: $0.87,95 \% \mathrm{Cl}: 0.41-1.86$ )[7]. However, this study was limited because the diagnoses of hypertension disorder and diabetes mellitus did not distinguish between different subtypes. In contrast, we found that gestational diabetes mellitus increased the risk of UR (aRR: 1.20, 95\% Cl: 1.031.41), but preeclampsia did not increase the risk of UR (aRR: $0.89,95 \% \mathrm{Cl}: 0.70-1.14$ ). Our finding is similar to results from two previous studies, where gestational diabetes increased the risk of UR (aOR: 5.78, 95\% Cl: 1.12-20.00)[19], and eclampsia was not related to the risk of UR (aOR: 0.08, 95\% Cl: $0.01-$ $0.71)[20]$.

Furthermore, we found that approximately six percent of women had two or more pregnancy complications. A previous study identified placenta previa as often occurring alongside placenta accreta, leading to a higher incidence of bleeding complications[38]. Moreover, placental abruption concurrently 
presents with preeclampsia in the same pregnancy, and these two complications have a similar pathogenesis, such as placental ischaemia[15]. The cooccurrence of preeclampsia and placental abruption was associated with worse maternal, foetal and neonatal outcomes (e.g., stillbirth/neonatal deaths)[39]. However, the combined effect of pregnancy complications on the risk of UR has been less closely studied. Our results indicate that having two or more pregnancy complications may increase the risk of UR (Model 2, aRR: 1.42, 95\% Cl: 1.14-1.77), but we failed to assess the effects of unique combinations due to small samples.

The pathophysiological mechanism underlying the relationship between pregnancy complications and the occurrence of UR remains unclear. The occurrence of UR may be directly or indirectly caused by a weak myometrium and excessive expansion of the uterine cavity. We speculate that the increased risk of UR may be due to pregnancy complications themselves through a specific mechanism. For example, UR from placenta percreta occurs because the placental villi invade the myometrium at the site of placental implantation (particularly at a previous scar site), resulting in UR[18]. In our study, we found that placenta percreta was still associated with an increased risk of UR in women without previous caesarean delivery. The possible reason for this might be the thinning of the uterus after multiple induced abortions, resulting in placental percreta leading to UR. In addition, we hypothesize that pregnancy complications may indirectly increase the occurrence of UR by increasing the occurrence of certain obstetric complications. Our results support the hypothesis that gestational diabetes mellitus in combination with LGA may increase the risk of UR. In our study, we found that the association between gestational diabetes mellitus without an LGA foetus and UR was 1.18-fold (Model 2, aRR: 1.18, 95\% Cl: 1.00-1.38), and the risk of UR was slightly larger in women with gestational diabetes mellitus and an LGA foetus (especially at 34-36 weeks gestational age). Our findings raise the possibility that UR may occur during late pregnancy among diabetic women, when excess foetal growth leads to excessive expansion of the uterine cavity. Future research is required to investigate the mechanism of pregnancy complications on the occurrence of UR.

UR often occurs before or during labour without warning. The risk of UR can increase in the presence of predisposing factors, such as a history of caesarean section and its related pregnancy complications. Clinicians may tend to focus on women with a history of caesarean delivery, ignoring those without such a history but with complications during pregnancy. Current guidelines developed by the American College of Obstetricians and Gynaecologists (ACOG) and Royal College of Obstetricians and Gynaecologists (RCOG) place a strong emphasis on the impact of vaginal trial delivery after caesarean section on UR[21, 22]. Unfortunately, there is currently a lack of guidelines for pregnant women with pregnancy complications to prevent the occurrence of UR. For example, current guidelines developed by the ACOG only recommend that women with gestational diabetes mellitus should be counselled regarding the risks and benefits of a scheduled caesarean delivery when the estimated foetal weight is $4,500 \mathrm{~g}$ or more[40]. In our study, we found that an LGA foetus (especially at 34-36 weeks gestational age) was associated with an increased risk of UR in women with gestational diabetes mellitus. Our findings suggest that controlling weight throughout pregnancy for women with gestational diabetes mellitus may play an important role in preventing the occurrence of UR. Additionally, we found that UR occurred only at term for women with placenta previa. Our findings support the recommendation that women with uncomplicated 
placenta previa should have a planned delivery at 36--37 weeks of gestation to avoid haemorrhage[41]. Moreover, evidence has shown that an accurate prenatal diagnosis and a standardized multidisciplinary team approach improve the pregnancy outcomes of women with placenta percreta[42]. Thus, standardized protocols for prenatal diagnosis and management of pregnancy complications should be established throughout pregnancy to reduce the occurrence of UR.

Our study has a number of strengths. First, we used common protocols to collect data through uniformly trained clinicians, so the data quality was high. Second, the large sample size allowed us to analyse the risk of UR with pregnancy complications in several subgroups. Third, we were able to adjust for several potential confounders (e.g., the number of previous caesarean deliveries, LGA, abnormal foetal presentation, advanced maternal age, multiple gravidities, coexisting comorbidity, etc. ).

We acknowledge some limitations within this study. First, there may be a possibility of the underreporting of pregnancy complications in our retrospective study. Additionally, some women with pregnancy complications may lost to follow up, when they abandoned treatment or were transferred to other nonmonitoring hospitals. However, some women with pregnancy complications may also be transferred from other non-monitoring hospitals into monitoring hospitals in the NMNMSS. Second, we were unable to obtain several variables that may have been related to the occurrence of UR, including information on the intended mode of delivery[43], the interval between this pregnancy and the last caesarean section[33], and the history of other uterine operations (e.g., myomectomy)[44]. Third, there was a lack of information on the duration and severity of pregnancy complications in our study, and there may be deviations in evaluating the impact of pregnancy complications on the risk of UR. Fourth, we did not distinguish between complete and partial UR[10], so we could not analyse the influence of pregnancy complications on the different types of UR. Therefore, the estimation of UR risk may be biased.

\section{Conclusions}

Our study identified that gestational diabetes mellitus, placental abruption, placenta previa and placenta percreta increased the risk of UR. An increased risk of UR before term birth was observed among women with gestational diabetes mellitus, placental abruption and placenta percreta. Furthermore, an LGA foetus increased the risk of UR in women with gestational diabetes mellitus, especially at 32 to 36 weeks gestation. Thus, physicians should provide early, better antenatal care and intervention for women with these high-risk pregnancy complications.

\section{Abbreviations}

NMNMSS: National Maternal Near Miss Surveillance System; UR: uterine rupture; LGA: large for gestational age; HELLP:haemolysis, elevated liver enzymes and low platelets; OGTT: oral glucose tolerance test; aRR: adjusted relative risk; Cl: confidence Interval; ACOG: American College of Obstetricians and Gynaecologists; RCOG: Royal College of Obstetricians and Gynaecologists 


\section{Declarations}

\section{Acknowledgements}

We thank the institutions and staff of the National Maternal Near Miss Surveillance System

\section{Authors' contributions}

JT, JL and JZ conceived the study. YM and YXX managed the dataset and PR C conducted the analyses. JT wrote the first draft of the manuscript. All authors provided intellectual input and contributed and approved the final manuscript.

\section{Funding}

This study has received funding by The National Key Research and Development Program of China (2018YFC1002200), The Sichuan province Key Research and Development Program of China (No. 2019YFS0530).

\section{Availability of data and materials}

The datasets used and/or analyzed during the current study are available from the corresponding author on reasonable request.

\section{Ethics approval and consent to participate}

This study was approved by the Ethics Review Committee of West China Second University Hospital, Sichuan University(Protocol ID: 2012008; date of approval: 3 March 2012), and conducted in accordance with the principles of the Declaration of Helsinki. Because of the retrospective design of this study, the Ethics Review Committee of West China Second University Hospital, Sichuan University has waived the requirement of the informed consent for this study.

\section{Consent for publication}

All authors have read and approved the content, and agree to submit it for consideration for publication in your journal.

\section{Competing Interests}

The authors declare no competing interests.

\section{References}

1. Kaczmarczyk M, Sparén P, Terry P, Cnattingius S. Risk factors for uterine rupture and neonatal consequences of uterine rupture: a population-based study of successive pregnancies in Sweden. BJOG: An International Journal of Obstetrics \& Gynaecology. 2007;114:1208-14. 
2. Motomura K, Ganchimeg T, Nagata C, Ota E, Vogel JP, Betran AP, et al. Incidence and outcomes of uterine rupture among women with prior caesarean section: WHO Multicountry Survey on Maternal and Newborn Health. Sci Rep. 2017;7:44093.

3. Zwart J, Richters J, Öry F, de Vries J, Bloemenkamp K, van Roosmalen J. Uterine rupture in the Netherlands: a nationwide population-based cohort study: Uterine rupture in the Netherlands. BJOG: An International Journal of Obstetrics \& Gynaecology. 2009;116:1069-80.

4. Tahseen S, Griffiths M. Vaginal birth after two caesarean sections (VBAC-2)-a systematic review with meta-analysis of success rate and adverse outcomes of VBAC-2 versus VBAC-1 and repeat (third) caesarean sections. BJOG. 2010;117:5-19.

5. Caughey AB, Shipp TD, Repke JT, Zelop CM, Cohen A, Lieberman E. Rate of uterine rupture during a trial of labor in women with one or two prior cesarean deliveries. Am J Obstet Gynecol. 1999;181:872-6.

6. Al-Zirqi I, Stray-Pedersen B, Forsén L, Vangen S. Uterine rupture after previous caesarean section: Uterine rupture. BJOG: An International Journal of Obstetrics \& Gynaecology. 2010;117:809-20.

7. Ronel D, Wiznitzer A, Sergienko R, Zlotnik A, Sheiner E. Trends, risk factors and pregnancy outcome in women with uterine rupture. Arch Gynecol Obstet. 2012;285:317-21.

8. Caughey AB, Cahill AG, Guise J-M, Rouse DJ. Safe prevention of the primary cesarean delivery. American Journal of Obstetrics and Gynecology. 2014;210:179-93.

9. Xia X, Zhou Z, Shen S, Lu J, Zhang L, Huang P, et al. Effect of a two-stage intervention package on the cesarean section rate in Guangzhou, China: A before-and-after study. PLoS Med. 2019;16:e1002846.

10. Al-Zirqi I, Stray-Pedersen B, Forsén L, Daltveit A-K, Vangen S. Uterine rupture: trends over 40 years. BJOG: Int J Obstet Gy. 2016;123:780-7.

11. Liang J, Mu Y, Li X, Tang W, Wang Y, Liu Z, et al. Relaxation of the one child policy and trends in caesarean section rates and birth outcomes in China between 2012 and 2016: observational study of nearly seven million health facility births. BMJ. 2018,:k817.

12. Getahun D, Oyelese Y, Salihu HM, Ananth CV. Previous Cesarean Delivery and Risks of Placenta Previa and Placental Abruption. Obstet Gynecol. 2006;107:771-8.

13. Daltveit AK, Tolla MC, Irgens LM. Cesarean Delivery and Subsequent Pregnancies. Obstet Gynecol. 2008;111:1327-34.

14. Hu H-T, Xu J-J, Lin J, Li C, Wu Y-T, Sheng J-Z, et al. Association between first caesarean delivery and adverse outcomes in subsequent pregnancy: a retrospective cohort study. BMC Pregnancy Childbirth. 2018;18:273.

15. Ananth CV. Ischemic placental disease: A unifying concept for preeclampsia, intrauterine growth restriction, and placental abruption. Semin Perinatol. 2014;38:131-2.

16. Brosens I, Pijnenborg R, Vercruysse L, Romero R. The "Great Obstetrical Syndromes" are associated with disorders of deep placentation. American Journal of Obstetrics and Gynecology. 2011;204:193201. 
17. Eshkoli T, Weintraub AY, Sergienko R, Sheiner E. Placenta accreta: risk factors, perinatal outcomes, and consequences for subsequent births. American Journal of Obstetrics and Gynecology. 2013;208:219.e1-219.e7.

18. Gupta N, Gupta A, Green M, Kang HS, Blankstein J. Placenta Percreta at 17 Weeks with Consecutive Hysterectomy: A Case Report and Review of the Literature. Case Reports in Obstetrics and Gynecology. 2012;2012:1-4.

19. Getahun WT, Solomon AA, Kassie FY, Kasaye HK, Denekew HT. Uterine rupture among mothers admitted for obstetrics care and associated factors in referral hospitals of Amhara regional state, institution-based cross-sectional study, Northern Ethiopia, 2013-2017. PLoS ONE. 2018;13:e0208470.

20. Vilchez G, Nazeer S, Kumar K, Warren M, Dai J, Sokol R. Contemporary epidemiology and novel predictors of uterine rupture: a nationwide population-based study. Arch Gynecol Obstet. 2017;296:869-75.

21. Royal College of Obstetricians and Gynaecogists., A, B, C, D, E, et al. Birth after Previous Caesarean Birth (Green-Top Guideline No. 45). 2015.

22. American College of Obstetricians and Gynaecologists., A, B, C, D, E, et al. ACOG Practice Bulletin No. 205: Vaginal Birth After Cesarean Delivery. Obstet Gynecol. 2019;133:e110-27.

23. Zhu J, Liang J, Mu Y, Li X, Guo S, Scherpbier R, et al. Sociodemographic and obstetric characteristics of stillbirths in China: a census of nearly 4 million health facility births between 2012 and 2014. The Lancet Global Health. 2016;4:e109-18.

24. Mclntyre HD, Catalano P, Zhang C, Desoye G, Mathiesen ER, Damm P. Gestational diabetes mellitus. Nat Rev Dis Primers. 2019;5:47.

25. Tarney CM, Whitecar P, Sewell M, Grubish L, Hope E. Rupture of an Unscarred Uterus in a Quadruplet Pregnancy. Obstet Gynecol. 2013;121:483-5.

26. Liang J, Mu Y, Li X, Tang W, Wang Y, Liu Z, et al. Relaxation of the one child policy and trends in caesarean section rates and birth outcomes in China between 2012 and 2016: observational study of nearly seven million health facility births. BMJ. 2018;360:k817.

27. Toppenberg KS, Block WA. Uterine rupture: what family physicians need to know. Am Fam Physician. 2002;66:823-8.

28. Murphy DJ. Uterine rupture. Curr Opin Obstet Gynecol. 2006;18:135-40.

29. Jauniaux E, Collins S, Burton GJ. Placenta accreta spectrum: pathophysiology and evidence-based anatomy for prenatal ultrasound imaging. Am J Obstet Gynecol. 2018;218:75-87.

30. Zhu L, Zhang R, Zhang S, Shi W, Yan W, Wang X, et al. Chinese neonatal birth weight curve for different gestational age. Zhonghua Er Ke Za Zhi. 2015;53:97-103.

31. Williams R. Using the Margins Command to Estimate and Interpret Adjusted Predictions and Marginal Effects. The Stata Journal. 2012;12:308-31. 
32. Abebe F, Mannekulih E, Megerso A, Idris A, Legese T. Determinants of uterine rupture among cases of Adama city public and private hospitals, Oromia, Ethiopia: a case control study. Reprod Health. 2018;15:161.

33. Al-Zirqi I, Daltveit AK, Forsén L, Stray-Pedersen B, Vangen S. Risk factors for complete uterine rupture. American Journal of Obstetrics and Gynecology. 2017;216:165.e1-165.e8.

34. Fadl HE, Östlund IKM, Magnuson AFK, Hanson USB. Maternal and neonatal outcomes and time trends of gestational diabetes mellitus in Sweden from 1991 to 2003. Diabetic Medicine. 2010;27:436-41.

35. Thisted DLA, Mortensen LH, Krebs L. Uterine rupture without previous caesarean delivery: a population-based cohort study. European Journal of Obstetrics \& Gynecology and Reproductive Biology. 2015;195:151-5.

36. Fitzpatrick KE, Kurinczuk JJ, Alfirevic Z, Spark P, Brocklehurst P, Knight M. Uterine Rupture by Intended Mode of Delivery in the UK: A National Case-Control Study. PLoS Med. 2012;9:e1001184.

37. Kinoshita T, Ogawa K, Yasumizu T, Kato J. Spontaneous rupture of the uterus due to placenta percreta at 25-weeks' gestation: a case report. J Obstet Gynaecol Res. 1996;22:125-8.

38. Usta IM, Hobeika EM, Abu Musa AA, Gabriel GE, Nassar AH. Placenta previa-accreta: Risk factors and complications. American Journal of Obstetrics and Gynecology. 2005;193:1045-9.

39. Han M, Liu D, Zeb S, Li C, Tong M, Li X, et al. Are maternal and neonatal outcomes different in placental abruption between women with and without preeclampsia? Placenta. 2019;85:69-73.

40. American College of Obstetricians and Gynaecologists., A, B, C, D, E, et al. ACOG Practice Bulletin No. 190: Gestational Diabetes Mellitus. Obstet Gynecol. 2018;131:e49-64.

41. Spong CY, Mercer BM, D'Alton M, Kilpatrick S, Blackwell S, Saade G. Timing of Indicated Late-Preterm and Early-Term Birth. Obstetrics \& Gynecology. 2011;118:323-33.

42. Shamshirsaz AA, Fox KA, Salmanian B, Diaz-Arrastia CR, Lee W, Baker BW, et al. Maternal morbidity in patients with morbidly adherent placenta treated with and without a standardized multidisciplinary approach. American Journal of Obstetrics and Gynecology. 2015;212:218.e1-218.e9.

43. Vandenberghe G, Bloemenkamp K, Berlage S, Colmorn L, Deneux-Tharaux C, Gissler M, et al. The International Network of Obstetric Survey Systems study of uterine rupture: a descriptive multicountry population-based study. BJOG: Int J Obstet Gy. 2019;126:370-81.

44. Getahun WT, Solomon AA, Kassie FY, Kasaye HK, Denekew HT. Uterine rupture among mothers admitted for obstetrics care and associated factors in referral hospitals of Amhara regional state, institution-based cross-sectional study, Northern Ethiopia, 2013-2017. PLoS ONE. 2018;13:e0208470.

\section{Figures}




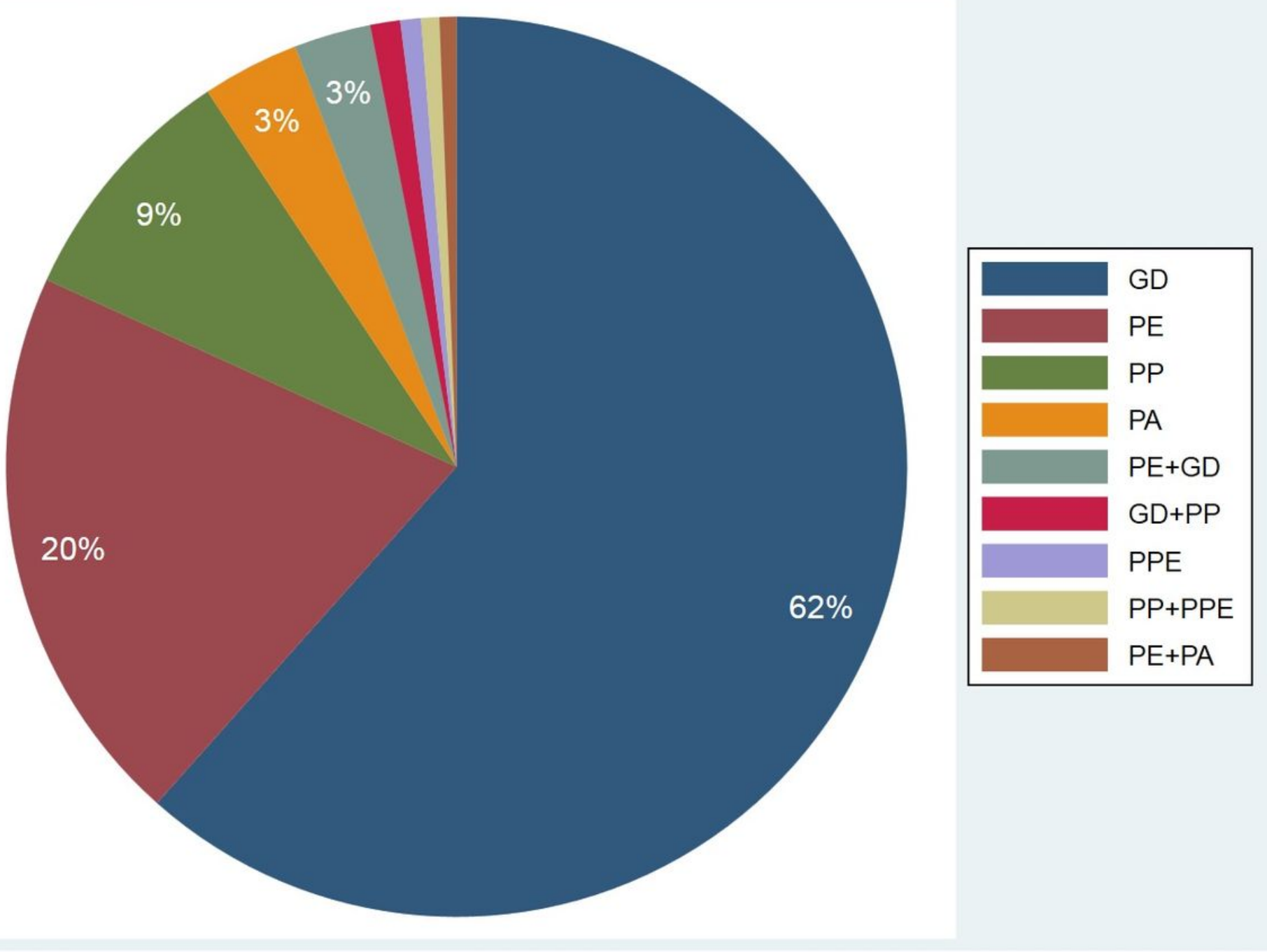

\section{Figure 1}

Type and percent of observed pregnancy complications among 875,245 pregnant women Note: We did show more than $0.3 \%$ of observed combinations of pregnancy complications among 885,087 pregnant women. Less than $1 \%$ of observed combinations of pregnancy complications were not labelled. GD: Gestational diabetes, PE: Preeclampsia, PP: Placenta praevia, PA: Placental abruption, PPE: Placenta percreta. 


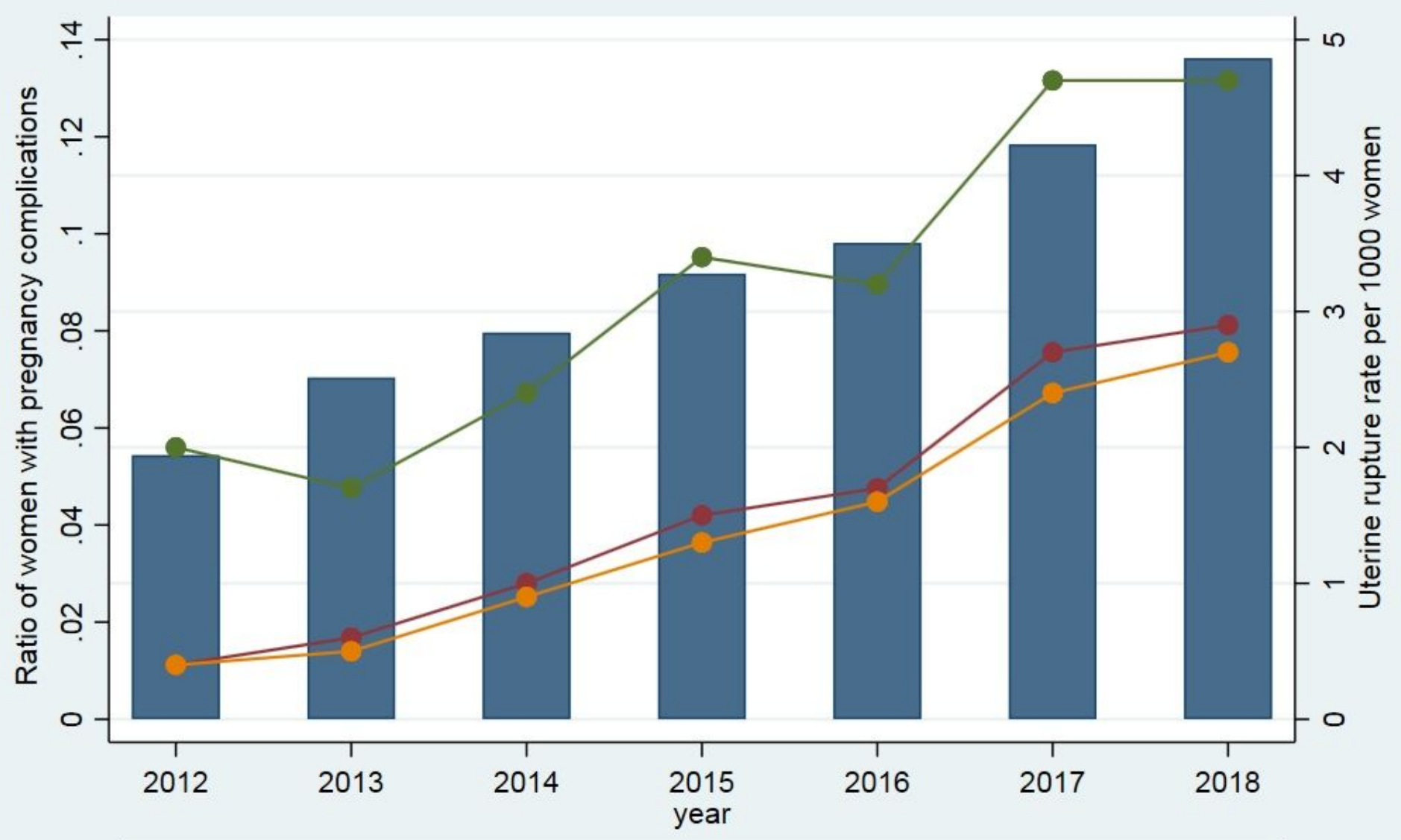

\section{Figure 2}

Uterine rupture rate and the ratio of women with pregnancy complications, China, 2012-2018 Note: The UR rate was weighted for the sampling distribution of the population covered by the Chinese National Maternal Near Miss Surveillance System. Ratio of women with pregnancy complications was the ratio of women with pregnancy complications to all women.

\section{Supplementary Files}

This is a list of supplementary files associated with this preprint. Click to download.

- Supplementaryfiles0906.docx 